\title{
Multiple Cerebral Infarcts in Patient with Moyamoya Disease
}

\author{
C.S. Casserly, A. Salmon, D.A. Ramsay, D.M. Pelz, S.P. Lownie, \\ M.J. Strong
}

Can J Neurol Sci. 2012; 39: 378-382

\section{Clinical Presentation - Dr. Casserly}

A 35-year old right-handed woman presented with an eight month history of progressive neurological symptoms that began with episodic twitching and paresthesiae of the right hand, lasting a few minutes. These episodes resolved with carbamazepine treatment. At the same time she noticed difficulty with word finding, memory and concentration, symptoms that she perceived as gradual and progressive over several months. Five months into her illness she developed numbness and tingling of the right side of her face and a severe headache on the left, associated with photophobia and phonophobia. In the tenth month of her illness, she reported a further deterioration in cognition and she had developed a non-fluent aphasia. She was assessed by a neurologist and admitted to hospital for further investigation.

Her past medical history includes Raynaud's phenomenon, migraine with aura, and a distant history of Bell's palsy. Her medication at the time of admission was limited to carbamazepine CR $200 \mathrm{mg}$ twice daily. There was no family history of migraine, cerebrovascular disease or psychiatric illness. Her maternal grandmother was diagnosed with Alzheimer's disease in her seventies.

At the time of admission her vital signs were normal, she was not hypertensive and she was not in acute distress. Her systemic examination was unremarkable. The patient was alert and oriented to person, place and time. Her language was moderately non-fluent and she had mild difficulty with naming. Her repetition of simple phrases, comprehension, reading and writing were intact. A formal mental status examination was not carried out. The cranial nerve findings were unremarkable and, in particular, there was no facial asymmetry. The motor examination revealed normal muscle bulk, tone and power, but there was a slight right pronator drift. The tendon reflexes were present and symmetrical and the plantar responses were flexor. There were no significant sensory findings, and co-ordination and gait were unremarkable.

\section{Case Discussion - Dr. Salmon}

This 35-year old patient presents with chronic progressive neurological dysfunction. Her symptoms and signs include headache on the left, difficulty with memory and concentration, non-fluent aphasia with preserved repetition, right facial paresthesias and subtle right upper-extremity weakness. Early episodic involuntary movements and paresthesias of the right hand had resolved with carbamazepine.

The aphasia, right facial paresthesias and right weakness localize to the left frontal lobe, including the inferior frontal gyrus, arm and hand areas of the primary motor area, and the facial area of the primary sensory cortex. The left-sided headache is also consistent with a left hemispheric process. The memory and concentration complaints are not precisely defined and therefore do not help with the process of localization. The involuntary movements of the right hand were probably partial seizures, also likely originating from the left primary motor area.

In summary, the progressive neurological findings localize to a moderately sized area of the left frontal lobe. There is a long differential diagnosis. Although this localization is commonly seen in middle cerebral artery strokes, the signs and symptoms had progressed gradually as opposed to the sudden or step-wise pattern that is expected for cerebrovascular disease, making this etiology unlikely.

Given the history of Raynaud's phenomenon, an autoimmune process should be considered. Although there were no findings on examination to support this suspicion- such as rash or arthritis - systemic lupus erythematosus, Sjogren's syndrome, antiphospholipid antibody syndrome and Hashimoto's encephalitis can all cause neuropsychiatric symptoms. Primary central nervous system (CNS) vasculitis can also lead to the combination of symptoms demonstrated by this patient. It should be noted that cerebral autoimmune disease tends to be more widely distributed, which does not appear to be the case in this patient. Given the focal seizures and the progressive nature of the neurological deficits, Rasmussen's encephalitis would have been a possibility at the outset but, because the seizures were

From the Department of Neurology (CSC, AS, MJS), Department of Pathology (DAR), Department of Neuroradiology (DMP), Department of Neurosurgery (SPL), London Health

Sciences Centre and the University of Western Ontario, London, Ontario, Canada.

Received September 26, 2011. Final Revisions Submitted November 9, 2011.

Reprint requests to: C.S. Casserly, c/o Department of Pathology (attn: Dr. Ramsay), University Hospital, London Health Sciences Center, 339 Windermere Road, London, Ontario, N6A 5A5, Canada. Email: courtney.casserly@londonhospitals.ca 
readily controlled with carbamazepine, this condition is no longer a consideration.

Other etiologies include neurosarcoidosis, a chronic infectious process such as syphilis, Lyme disease, HIV encephalitis, a brain tumor, or paraneoplastic encephalitis. Primary CNS lymphoma would have caused a more rapid progression of symptoms than the patient manifested.

As the patient also has a history of migraine headaches, a positive family history may have suggested either CADASIL or MELAS, both rare causes of headache and progressive neurological dysfunction. These conditions are unlikely in the absence of a similar family history and the lack of systemic manifestations of mitochondrial disease.

Initial investigations should include: a complete blood count and differential; measurement of electrolytes; liver and kidney function tests; TSH; an ACE level; a screen for autoimmune disease and vasculitis; VDRL, HIV and Lyme serology; and a sampling of cerebrospinal fluid (CSF). Magnetic resonance imaging (MRI) of the brain and an electroencephalogram (EEG) should be completed.

\section{Clinical Presentation - Dr. Casserly (INITIAL INVESTIGATIONS)}

The following studies were unremarkable: electrolytes; urea; creatinine; blood clotting parameters (INR, PTT, platelets); erythrocyte sedimentation rate; C-reactive protein; rheumatoid factor; complement; antinuclear antibody [weakly positive]; anti-extractable nuclear antigen; anti-phospholipid antibody; anti-double stranded DNA; anti-neutrophil cytoplasmic antibodies; immunoglobulin levels; cryoglobulins; hepatitis B and C; Lyme disease; HIV [VDRL was not carried out]). No endocrine abnormalities were found (i.e. serum cortisol, thyroid stimulating hormone, leutinizing hormone, follicle stimulating hormone, prolactin, beta-human chorionic gonadotrophin were normal). She was slightly anemic (hemoglobin $101 \mathrm{~g} / \mathrm{L}, \mathrm{MCV}$ $90.9 \mathrm{fL}$ ) and there was a marginal increase in the white cell count (WBC $10.310-9 / \mathrm{L}$ ). The CSF protein, glucose and cell count were unremarkable.

The EEG contained a striking focal abnormality of the left cerebral hemisphere. Specifically, the left hemisphere generated diffuse delta waves (graded dysrhythmia grade II), while the right hemisphere had an appropriate alpha rhythm. No definite spikes were identified. The electrographic interpretation was consistent with a left hemispheric structural abnormality.

The first computed tomogram (CT) scan of her head was performed four months into her illness and revealed an area of low density in the deep white matter of the left posterior frontal centrum semiovale; ill-defined white matter changes were also apparent rostral to the frontal lesion and throughout the left cerebral hemisphere. The brain MRI, carried out six months later when she was admitted to hospital, was characterized by multiple areas of diffusion restriction on diffusion weighted imaging in left cerebral hemisphere (Figure 1A). There was no significant mass effect or enhancement with administration of gadolinium. Examination of the MR angiogram and conventional digital subtraction angiogram of the head and neck revealed significant narrowing of the internal carotid arteries and middle cerebral arteries bilaterally, with minor changes also noted in the small vessels (Figure 1B, 1C). There were collateral vessels from the posterior circulation to both the ACA and MCA, most evident on the left anterior-posterior (AP) digital subtraction angiogram (Figure 1D). A CT perfusion study of the brain revealed a marked decrease in cerebral blood flow in both cerebral hemispheres with a corresponding increase in mean transit time, particularly in the left cerebral hemisphere; there was also a marked decrease in cerebral blood volume in the right posterior temporal and parietal lobes - these findings were interpreted by neuroradiology as suggestive of imminent infarction of the left hemisphere. A subsequent angiogram, performed approximately one month following admission to hospital, demonstrated complete occlusion of the right internal carotid artery (ICA) (Figure 1E).

\section{Further Case Discussion - Dr. Salmon}

The MRI imaging confirms a left hemisphere process, but also reveals bilateral carotid arterial disease. The imaging

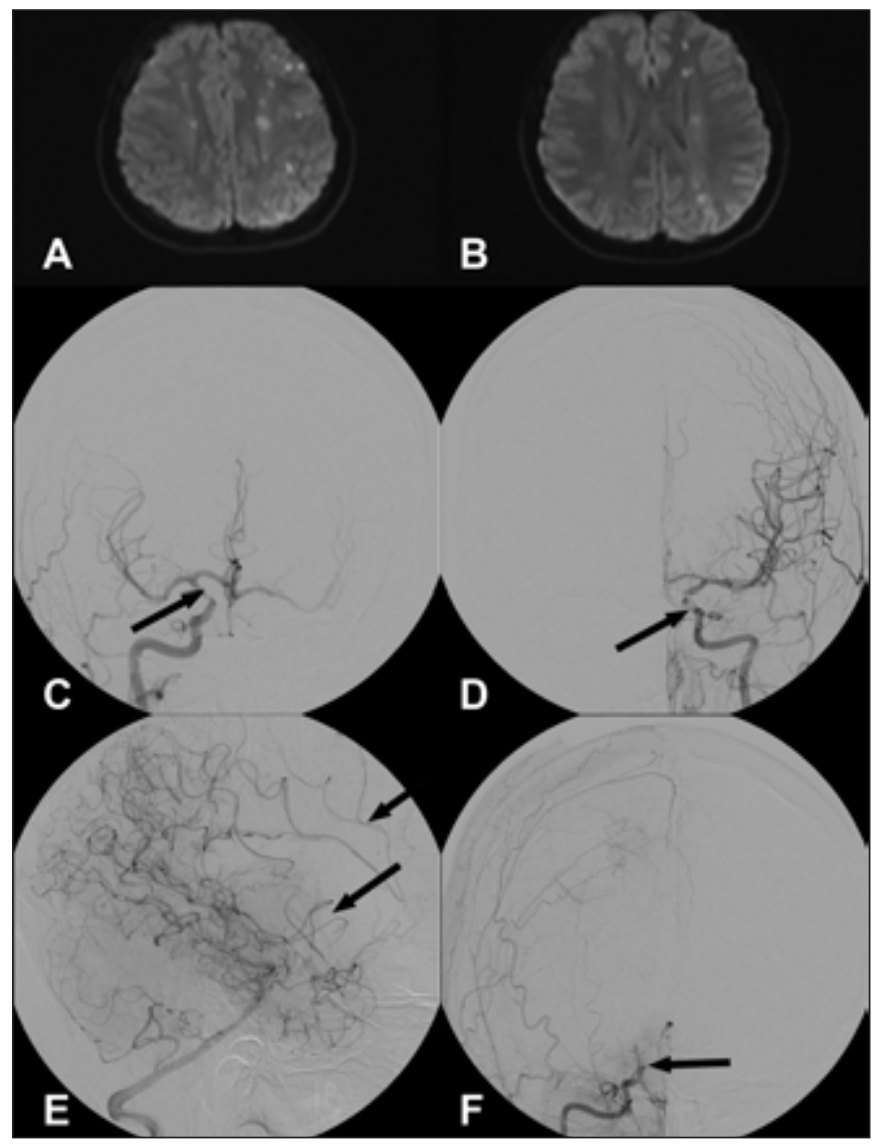

Figure 1: A and B) Axial MRI head, diffusion weighted images, demonstrating multiple areas of diffusion restriction left cerebral hemisphere. C and D) Digital subtraction angiogram (DSA) of the head, right and left anterior-posterior $(A P)$ views respectively, demonstrating significant narrowing of the internal carotid arteries (ICA) bilaterally. E) DSA of the head, lateral view of left vertebral artery, demonstrating collateral vessels from the posterior circulation to both the ACA and $M C A . F)$ Subsequent DSA head, right AP view, performed 1 month after hospitalization, demonstrating complete occlusion of the right ICA. 
findings are consistent with a combination of acute and chronic infarcts and exclude a neoplasm. The MRI and angiographic findings of multiple strokes of various ages, associated with narrowing and beading of small arteries, are typical of vasculitis, whereas the narrowing of the internal carotid and middle cerebral arteries and the collateralization are found in Moyamoya disease. The serological investigations and CSF findings exclude autoimmune and infectious causes of both vasculitis and Moyamoya disease. Primary CNS vasculitis may be associated with non-specific CSF abnormalities ${ }^{1}$, such as mildly elevated WBCs or protein, but the absence of these findings, as in this case, does not exclude this diagnosis even though the involvement of large arteries would be unusual in vasculitis. Immunosuppressive therapy should be considered and a brain biopsy may be helpful to confirm the diagnosis.

\section{Clinical Presentation - Dr. Casserly (SUMMARY)}

The patient had been admitted to hospital with a working diagnosis of CNS vasculitis. A brain biopsy was performed but was unremarkable. She was continued on carbamazepine, and prednisone and cyclophosphamide treatment was started.

The angiography was repeated approximately one month after admission to hospital. The right internal carotid artery had become occluded and the right hemisphere was being perfused by a posterior collateral circulation. There was high grade stenosis of the left internal carotid artery with poor perfusion in left ICA territory, which was confirmed by CT cerebral perfusion studies (described above in 'Initial Investigations'). The patient remained stable in the ICU with volume expansion and aggressive induced hypertensive therapy using multiple inotropes to maintain cerebral perfusion.

Over the next several weeks she developed confusion, a worsening non-fluent aphasia, a right facial droop and worsening right arm weakness. Her symptoms worsened each time attempts were made to wean her from the inotropic support. She then underwent an angioplasty of the left ICA, which was complicated by an iatrogenic left ICA dissection. Postoperatively, there was radiographic evidence of improved blood flow through the left ICA.

One week after the angioplasty the patient's level of consciousness decreased, her aphasia worsened and she had a generalized tonic-conic seizure. The MRI now revealed a watershed infarct of the right cerebral hemisphere. The decision was made to perform an arterial bypass graft between the left superficial temporal artery and the angular branch of the left middle cerebral artery (EC-IC bypass). This was performed on May 6, 2011. Intraoperative angiography showed a remarkable degree of vasospasm within the territory of the operated vessel but also in the more proximal extracranial circulation supplying the brain. Within two days the patient declined further with an extensive left hemisphere stroke. Angiography showed a patent bypass, but the intracranial ICA had gone on to occlude completely. Decompressive craniectomy and duraplasty were performed on May 8, 2011. Despite this the patient did not improve and with CT evidence of stroke in multiple vascular territories she died 11 days post bypass.

\section{Case Discussion - Dr. Salmon \\ (Final Clinical Comments)}

A negative brain biopsy does not exclude cerebral vasculitis because the inflammation is often patchy and may not be present in the sampled tissue. If the clinical suspicion for vasculitis remains, patients should be treated with immunosuppressive agents, as was carried out in this case. However, the continued deterioration in her condition over a relatively short period despite immunosuppressive therapy, including the progression of her carotid arterial stenosis, favors a diagnosis of Moyamoya disease. Ongoing transient ischemic attacks and cerebral infarcts are not uncommon complications following EC-IC bypass in patients with Moyamoya disease, either secondary to the disease or as an operative complication.

\section{Neuropathological Diagnosis - Dr. Ramsay}

The general autopsy revealed no specific features. In particular there was no evidence of vasculitis, atheroma, atherosclerosis or hypertension (heart weight $379 \mathrm{~g}, 95 \%$ confidence limits for women weighing $80 \mathrm{~kg}=202 \mathrm{~g}$ to $435 \mathrm{~g}$ ). Secondary findings included right lower lobe bronchopneumonia and marked pulmonary congestion.

There was a recent left craniotomy and an epidural hematoma related to the operative procedure. The brain weight was $1624 \mathrm{~g}$. There was severe brain swelling, including transcraniotomy herniation and bilateral moderate uncal herniation (more marked on the right than the left).

Examples of the gross findings are illustrated in Figure 2. Large areas of the left hemisphere were soft, friable and cavitated, owing to the effects of the brain swelling and to extensive ischemic changes, including multiple small infarcts of various ages and more extensive and widespread recent ischaemic neuronal necrosis. The left cerebral hemispheric infarction can be ascribed to the occlusion of the left internal carotid artery, which had been demonstrated by neuroimaging techniques six days before her death. Widespread hypoxicischaemic neuronal changes were also apparent in the right cerebral hemisphere, which, despite the MRI findings, was grossly relatively intact; there was also an acute infarct, hours to a few days old, in the right putamen and an older infarct, days to weeks old, in the anterior limb of the right internal capsule. The relative lack of 'infarcts' conforming to specific vascular territories, particularly in the right cerebral hemisphere, presumably reflects the effectiveness of the collateral circulation that developed as a result of the slow, progressive narrowing of the carotid arteries.

The arrangement of the circle of Willis was unremarkable. There were similar and distinctive histological abnormalities in the interosseous carotid arteries and the intracranial vessels, predominantly those of the anterior circulation. These changes included eccentric and focal intima thickening, pronounced undulation and hypertrophy of the internal elastic lamina (IEL), layered reduplication of the IEL, and segmental atrophy of the arterial media. The intimal changes included proliferated myofibroblasts, collagenisation and deposition of various amounts of alcianophilic proteoglycans, a pattern that could be described as 'nodular intimal fibroproteoglycanosis' (Figure 3A). There was bilateral focal mixed fibrotic and thrombotic occlusion of both intracranial internal carotid arteries (figure 


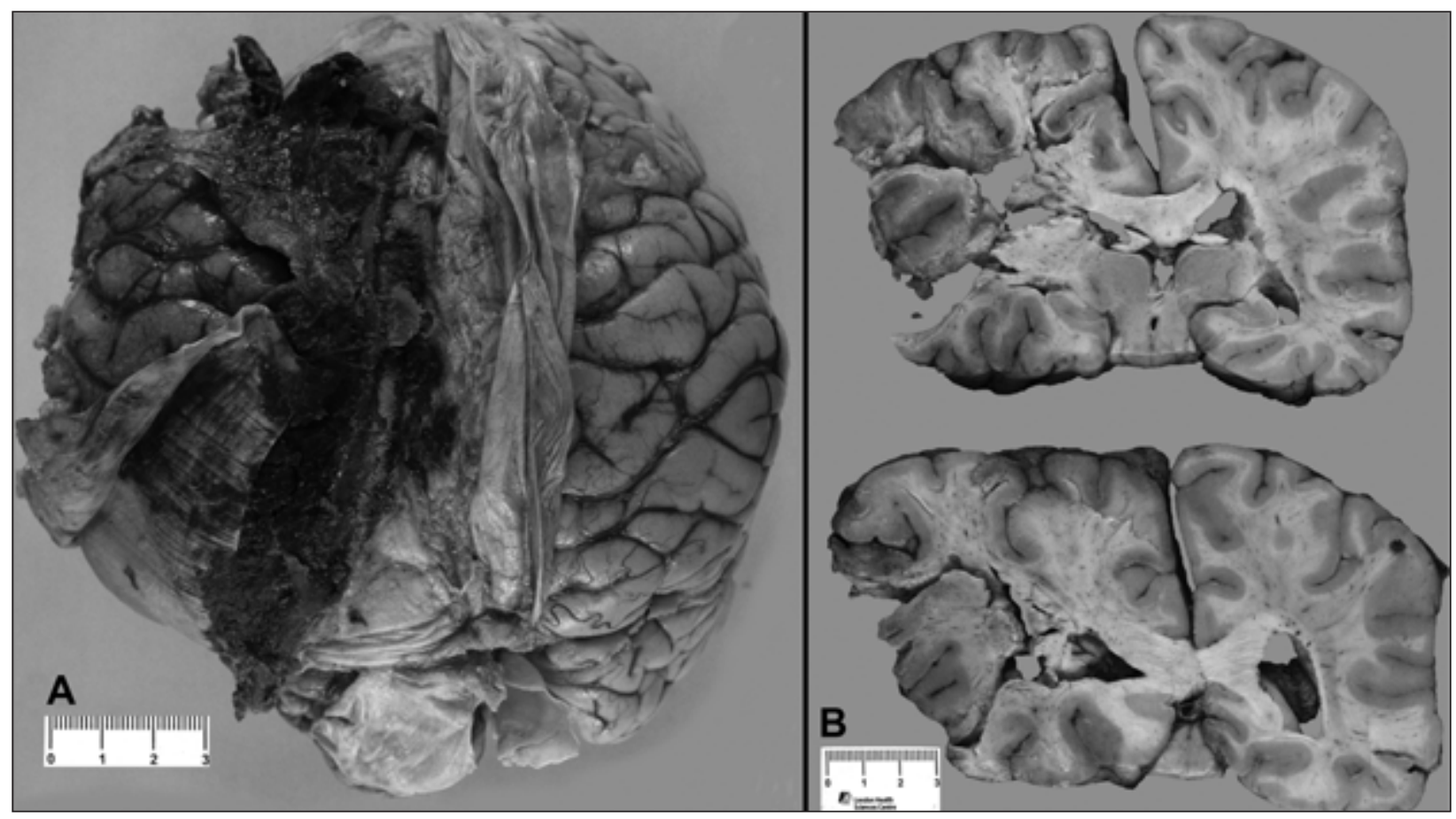

Figure 2: (A) This panel is a view of the brain vertex with the dura attached (the right dura has been reflected to the midline). There is a moderate amount of epidural blood, secondary to the craniotomy procedure, and marked transcraniotomy herniation. There is also effacement of the sulci and flatness of the gyri on the right, indicating diffuse brain swelling. (B) This panel shows two coronal slices through the cerebral hemispheres to illustrate the duskiness and fragmentation in the left cerebral hemisphere, involving the left middle and anterior cerebral artery territories, and the secondary left and right subfalcine herniation. Despite significant involvement of the right internal carotid artery by the disease process, the lack of grossly discernible infarcts both in the slices and elsewhere is remarkable.

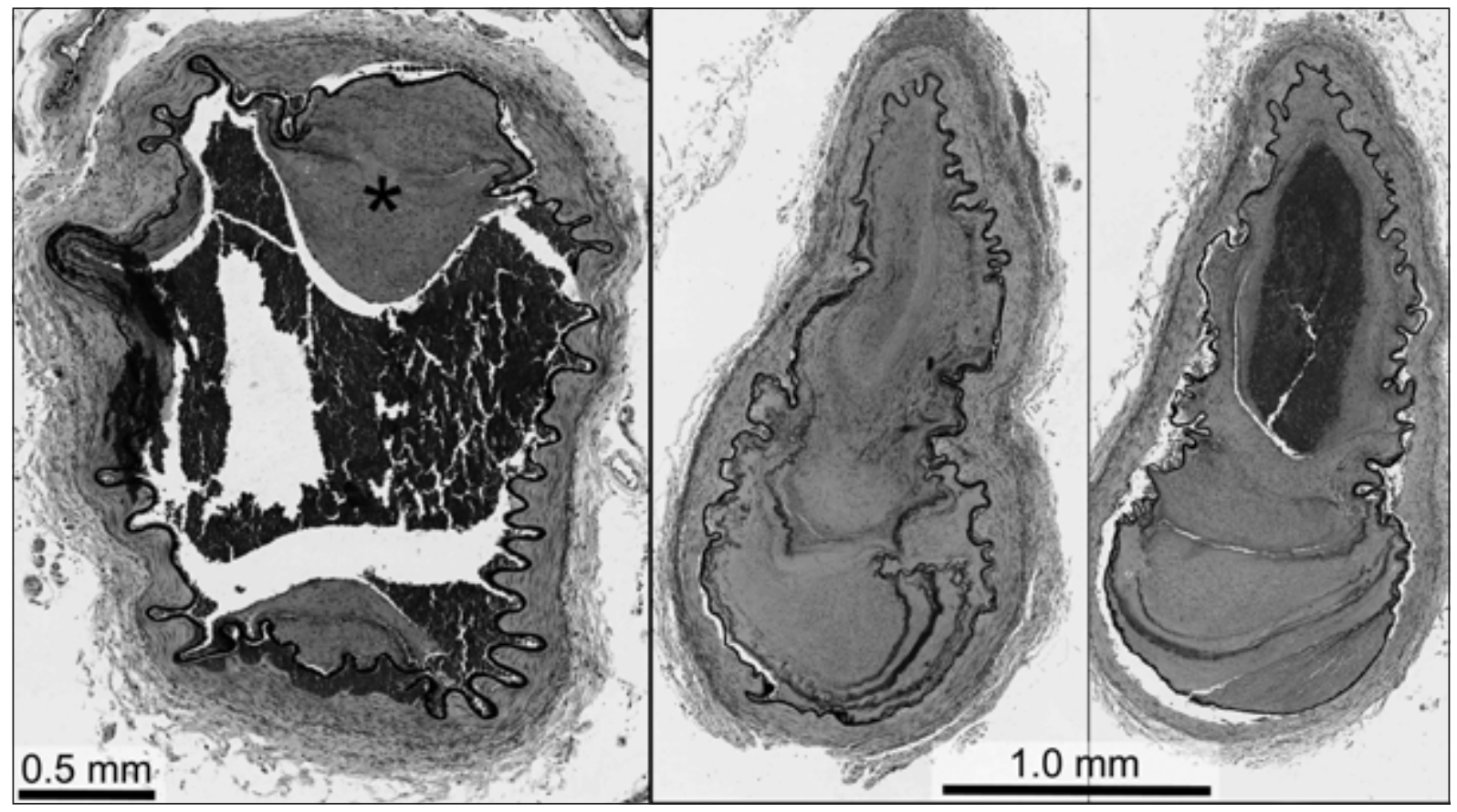

Figure 3:(Movat's trichrome): (A) This panel is from the left middle cerebral artery. It shows nodular proliferation of intimal myofibroblasts and related connective tissue substance (asterisks), an abnormality that was seen in many arterial profiles ('nodular intimal fibroproteoglycanosis'). (The apparent dissection in the lower part of the photomicrograph is an artifact.). (B) The paired panels on the right illustrate the established fibrotic occlusion of the left internal carotid artery and, further distally, an organizing thrombus. The lack of neovascularization and hemosiderosis in the fibrotic tissue in the lower third of each of these two panels marks the site of the original and evolving intimal fibroproteoglycanosis. 
3B). The EC-IC bypass anastomosis was intact but the bypass vessel was occluded by an organizing thrombus. Recent emboli were noted in the small cerebral vessels. The left internal carotid dissection was not identified. There was no evidence of atheroma or vasculitis. The extracranial carotid arteries were normal.

The neuropathological examination has therefore revealed a peculiar distribution of otherwise nonspecific vasculopathic findings with secondary ischaemic brain injury related to the progressive narrowing and ensuing acute thrombotic vascular occlusion of the cerebral blood vessels and of the EC-IC bypass graft. Moyamoya disease is a clinicopathological diagnosis (of exclusion), corresponding to a condition that is characterized radiologically by "...stenosis ... in the distal internal carotid artery [that] often also involves the proximal anterior and middle cerebral arteries" (2) and histologically by the finding "in the carotid terminations... [of] fibrocellular thickening of the intima..." (3). Accordingly, since both these criteria are found in the present case and because there is no other explanation for the patient's condition the neuropathological diagnosis is 'consistent with Moyamoya disease'.

\section{Moyamoya Disease - Dr. CASSERly Summary And Review}

Moyamoya disease is a chronic progressive vascular disease of the central nervous system, characterized by narrowing of the large arteries of the brain and prominent collateral circulation. 'Moyamoya' means "puff of smoke" in Japanese, which refers to the 'smokey' angiographic appearance of the collateral vessels.

Moyamoya disease was first described in Japan in 1957 and has a higher incidence among Japanese and Asian populations ${ }^{1-}$ 5 . There is a slight predilection for women $(2: 1)^{5}$, and a biphasic age distribution of $9-15 \mathrm{yrs}$ and $40-49 \mathrm{yrs}^{1-4}$. The etiology is unknown but there is likely a genetic component because $10 \%$ $15 \%$ of affected individuals have a family history of the disease, and concordance rates are high among siblings $(3-6 \%)^{6-8}$. The incidence world-wide is estimated to be $0.3-0.9 / 100,000$ and the prevalence $3.2-10.5 / 100,000^{4,5}$. The disease is rare in North America and so there is only limited population-based epidemiological data. The largest population based study used hospital discharge databases for California and Washington State and reported an incidence of $0.086 / 100,000$ person/year ${ }^{8}$. The estimated mortality rates range from $2-16 \%{ }^{1,3,7}$.

The diagnosis of Moyamoya disease is based on the characteristic angiographic appearance, including distal narrowing/occlusion of the internal carotid arteries and circle of Willis and multiple cerebral infarcts. A collateral meshwork of overgrown and dilated small arteries that branch off the circle of Willis is an inconstant hallmark of the disease ${ }^{2,3,9}$. The MRA diagnostic criteria proposed by a Japanese research committee include: [1] Stenosis or occlusion of the terminal portion of the internal carotid artery and at the proximal portion of the anterior and middle cerebral arteries on MRA; [2] abnormal vascular networks in the basal ganglia; and [3] bilateral findings ${ }^{5}$.

This condition can be sub-classified into a primary Moyamoya disease or to a secondary Moyamoya syndrome. The best established secondary cause of Moyamoya syndrome is head and neck radiation; other associated conditions include sickle cell anemia, neurofibromatosis type I, and Down's syndrome and, rarely, giant cervicofacial hemangiomas, hyperthyroidism, renal artery stenosis and congenital cardiac abnormalities ${ }^{2}$.

The implications of this diagnosis for the patient's family are unclear. Though Moyamoya disease does appear to have a genetic predisposition, the gene(s) involved have not yet been elucidated. At present, no screening angiography is recommended for family members.

The major differential diagnosis for this presentation of multiple cerebral infarcts in a young woman includes atherosclerosis, systemic autoimmune disease and vasculitis. In retrospect, the significant involvement of the large vessels of the anterior circulation, the presence of extensive collaterals on imaging and the lack of a response to immunosuppressive therapy to treat supposed vasculitis may have raised the possibility of Moyamoya disease. Nevertheless, an EC-IC bypass was performed, which is ultimately the treatment of choice for Moyamoya disease ${ }^{10,11}$. Whether or not earlier recognition and surgical treatment would have affected the course of the disease is uncertain.

In conclusion, as exemplified by this case, Moyamoya disease is difficult to diagnose in life, it may progress rapidly and its mortality rate may be high despite intensive treatment ${ }^{1,3,7}$.

\section{REFERENCES}

1. Kraemer M, Berlit P. Primary central nervous system vasculitis and moyamoya disease: similarities and differences. J Neurol. 2010; 257(5):816-19.

2. Smith MR, Smith ER. Moyamoya disease and moyamoya syndrome. N Engl J Med. 2009;360(12):1226-37.

3. Kuroda S, Houkin K. Moyamoya disease: current concepts and future perspectives. Lancet Neurol. 2008;7(11):1056-66.

4. Goto Y, Yonekawa Y. Worldwide distribution of moyamoya disease. Neurol Med Chir (Tokyo). 1992;32(12):883-6.

5. Fukui M. Current state of study on moyamoya disease in Japan. Surg Neurol. 1997;47(2):138-43.

6. Inoue TK, Ikezaki K, Sasazuki T, Matsushima T, Fukui M. Linkage analysis of moyamoya disease on chromosome 6. J Child Neurol. 2000;15(3):179-82.

7. Roder C, Nayak NR, Khan N, Tatagiba M, Inoue I, Krischek B. Genetics of moyamoya disease. J Hum Genet. 2010;55(11): 711-16.

8. Uchino K, Johnson SC, Becker KJ, Tirschwell DL. Moyamoya disease in Washington State and California. Neurology. 2005;65 (6):956-8.

9. Scott RM, Smith ER. Introduction: moyamoya disease. Neurosurg Focus. 2009;26(4):E1

10. Karasawa J, Kikuchi H, Furuse S, Kawamura J, Sakaki T. Treatment of moyamoya disease with STA-MCA anastomosis. J Neurosurg. 1978;49(5):679-88.

11. Suzuki J, Kodama N. Moyamoya disease--a review. Stroke. 1983; 14(1):104-9. 